\title{
Waveguide Structures with Improved Cut-off Frequency and Bandwidth
}

\author{
Ramakrushna Panigrahi, Siba Prasad Mishra, P.V.Y.Jayasree
}

\begin{abstract}
The wave transmission characteristics of rectangular, double-ridge, trapezoidal-ridge and anti-trapezoidal ridge waveguides are analyzed using the finite-element method. The cut-off wavelength and attenuation of these waveguides are calculated. The result shows that anti-trapezoidal ridge waveguides perform better than rectangular, double-ridge and trapezoidal-ridge waveguides. The variation of bandwidth and attenuation with respect to change in the angle of physical ridge structures has been studied while migrating from rectangular to anti-trapezoidal ridge structures.
\end{abstract}

Keywords - waveguides; cut-off frequency; attenuation; rectangular, double-ridged; trapezoidal; anti-trapezoidal.

\section{INTRODUCTION}

\section{R} cut-off frequency and better bandwidth characterizes at dominant mode [1]. Ridge rectangular waveguides are also used as low impedance broadband structures. Due to wide frequency bandwidth, low dominant mode cutoff frequency and the characteristic impedance is smaller than rectangular waveguide structures ridge waveguides are used in many applications like phase shifters, duplexers, ridge waveguide transmitters and ridge bandwidth filters [1-3]. For many years various methods have been proposed for the analysis of rectangular, ridge waveguides. In recent studies various method been proposed for the study of trapezoidal waveguides. The study and analysis of trapezoidal waveguides show that in many way trapezoidal waveguides performs better than rectangular and ridge waveguides [5-7]. In ridge and trapezoidal waveguide design, the position and dimension of ridges play a major role in its performance and signal transmission in an optimized way [4]. The dimensions of ridges are optimized to minimize dominant mode cut-off frequency while the increase of the dominant mode bandwidth [1-2]. A popular method for determining the cut-off frequency, attenuation and bandwidth is Finite Element Method which is been used for a long time and it provides the accurate result.

Aim of this analysis is to develop a waveguide which will have better performance than existing all waveguide

Revised Manuscript Received on December 13, 2019.

* Correspondence Author

Ramakrushna Panigrahi*, Dept. of Electrical Electronics and Communication Engineering, GIT, GITAM University, Visakhapatnam, INDIA. Email: ramakrushna.panigrahi@gitam.edu

Siba Prasad Mishra, Dept. of Electrical Electronics and Communication Engineering, GIT, GITAM University, Visakhapatnam, INDIA. Email: sibaprasad.mishra@gitam.edu

P.V.Y Jayasree, Dept. of Electrical Electronics and Communication Engineering, GIT, GITAM University, Visakhapatnam, INDIA. Email: jayasree.pappu@gitam.edu structures in WR90 waveguide category. By varying the angle of ridge starting from an existing popular plane rectangular WR90 waveguide to double ridge waveguide structure which is migrating through the number of trapezoidal structures and then through series of anti-trapezoidal structures, the dominant mode cut-off frequencies and attenuation characteristics are analyzed in detail in this paper. All these simulations are done using HFSS.

\section{DESCRIPTION OF RECTANGULAR, DOUBLE RIDGE AND TRAPEZOIDAL WAVEGUIDES}

Consider a rectangular waveguide structure WR90 of length $\mathrm{L}$ with dimension $\mathrm{a} \times \mathrm{b} \times \mathrm{L}$ as shown in fig. 1 , where the cavity is filled with air and the wall of the waveguide are composed of perfect conducting material.

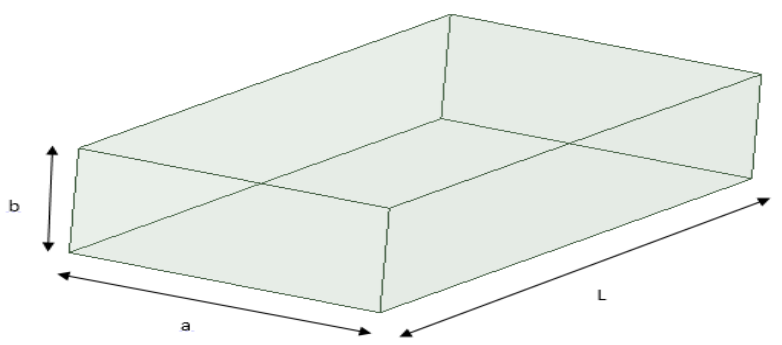

Fig. 1. A typical cross section of rectangular waveguide

The corresponding ridge waveguide structure WR90 of same length $\mathrm{L}$ with outer dimension $\mathrm{a} \times \mathrm{b} \times \mathrm{L}$ and having ridge dimension $\mathrm{c} x \mathrm{~d}$ is as shown in fig. 2 .

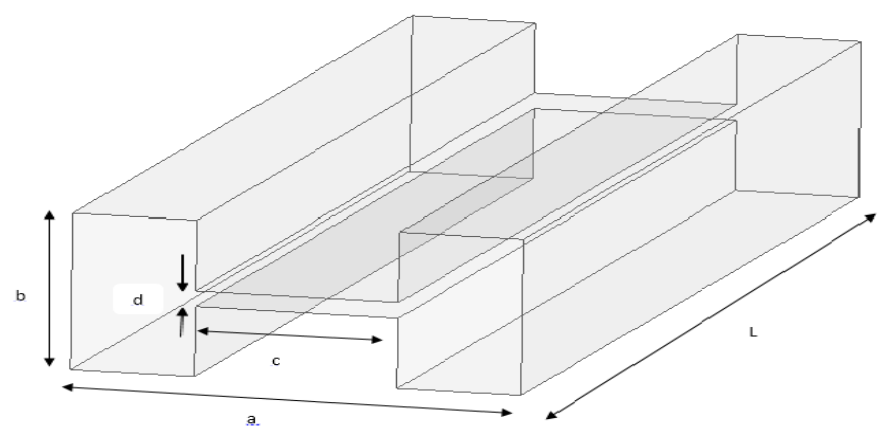

Fig. 2. A typical cross section of double ridge waveguide

For the same outer dimension, a $\mathrm{x}$ b $\mathrm{x}$ L of WR90 waveguide by keeping ridge gap ' $d$ ' constant and making arm 'c' variable as shown in fig. 3 we will obtain a series of trapezoidal and anti-trapezoidal waveguides where the angle of ridges are only variable between two extremes.

The angle variation will result in a number of trapezoidal waveguides as shown in fig. 4

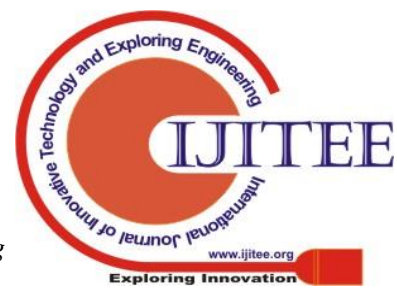


from which only standard angles considered here for calculation and observation purpose. All these waveguides are prepared with the same material, and under the same constraint, same boundary conditions and same excitations. The series of waveguides which results because of angle variations are considered for analysis purpose.

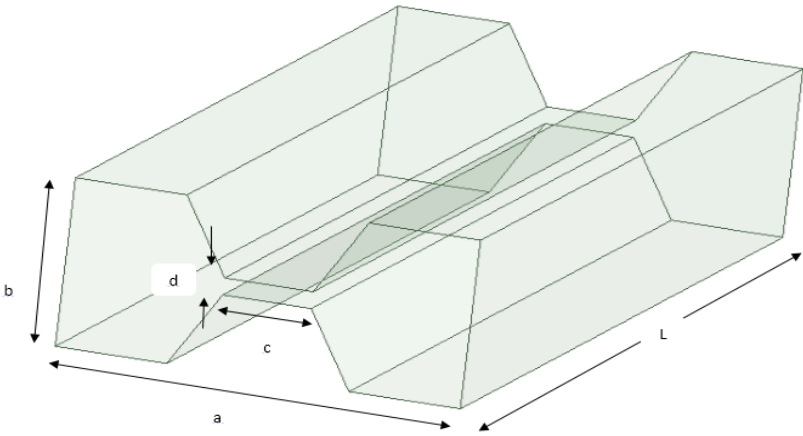

Fig. 3. Cross section of trapezoid double ridge waveguide

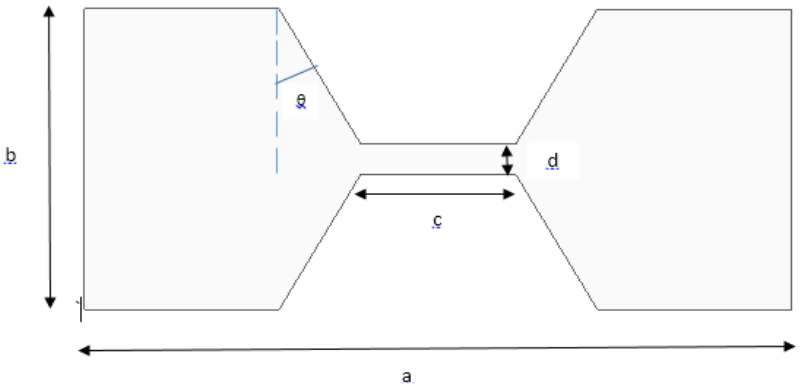

Fig. 4. Front View of trapezoid double ridge waveguide

\section{RESULT ANALYSIS}

The analysis of these waveguides is being done for S, C, X, $\mathrm{Ku}, \mathrm{K}$ and $\mathrm{Ka}$-band $(2 \mathrm{GHz}-30 \mathrm{GHz})$. Waveguide dimensions are $\mathrm{a} / \mathrm{b}=2.25, \mathrm{~b} / \mathrm{d}=10, \mathrm{c}$ and theta are variables.

The waveguide modal analysis is done for the first five modes. The double ridge trapezoidal waveguides results obtained by this method are compared with that of rectangular and double ridge waveguides. The rectangular and double ridge waveguides structures are being simulated and analyzed by many so far. In this paper, the modal analysis of trapezoidal and anti-trapezoidal structures are discussed.

On the basis of some standard structures, we studied the cut-off frequency of waveguide structures and the results showing their cut-off frequencies for the first five modes are as shown in Fig. 5 - 10.

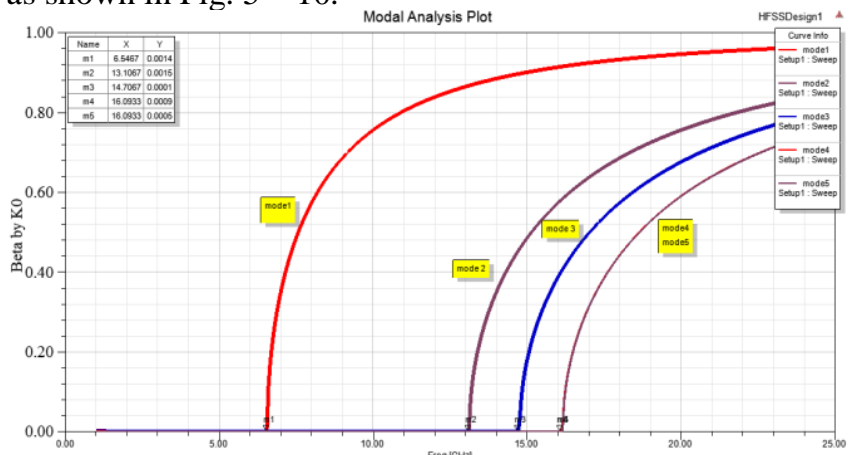

Fig. 5. First Five Modes of Rectangular Waveguide $(\theta=$ 900)

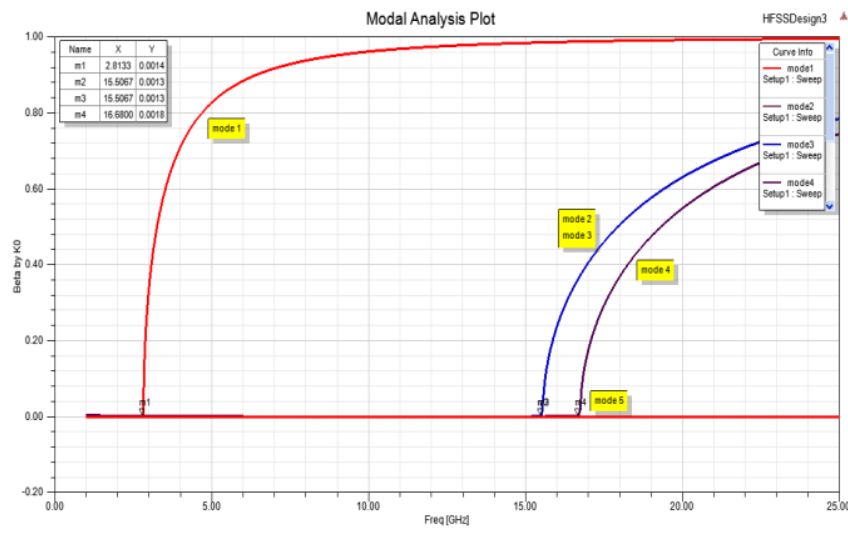

Fig. 6. First Five Modes of Trapezoidal Waveguide $(\theta=$ 300)

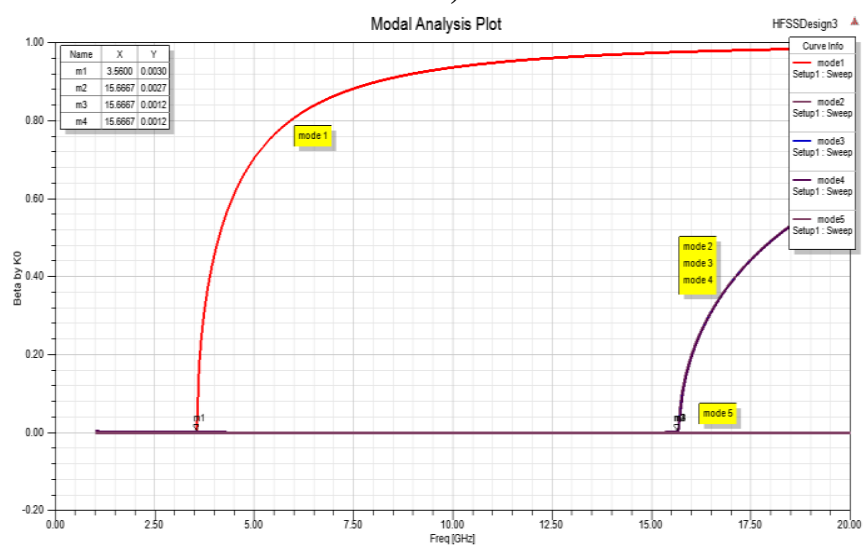

Fig. 7. First Five Modes of Trapezoidal Waveguide $(\theta=$ 450)

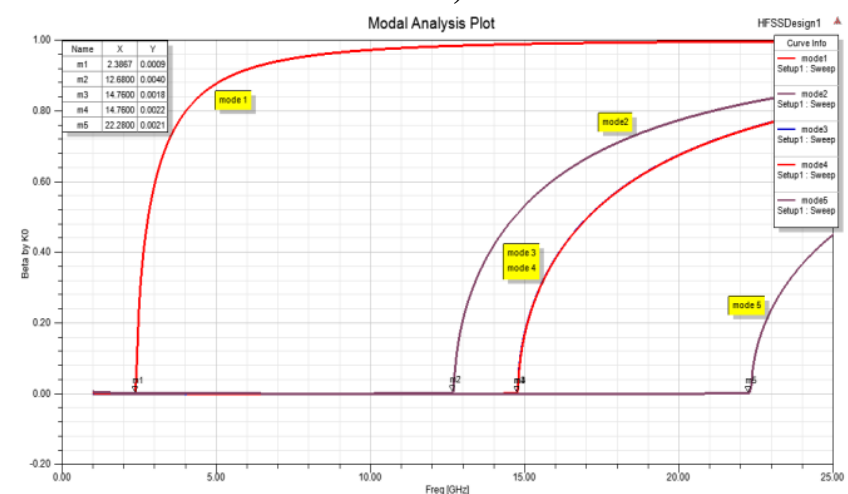

Fig. 8.First Five Modes of Double Ridge Waveguide $(\theta=$ 00)

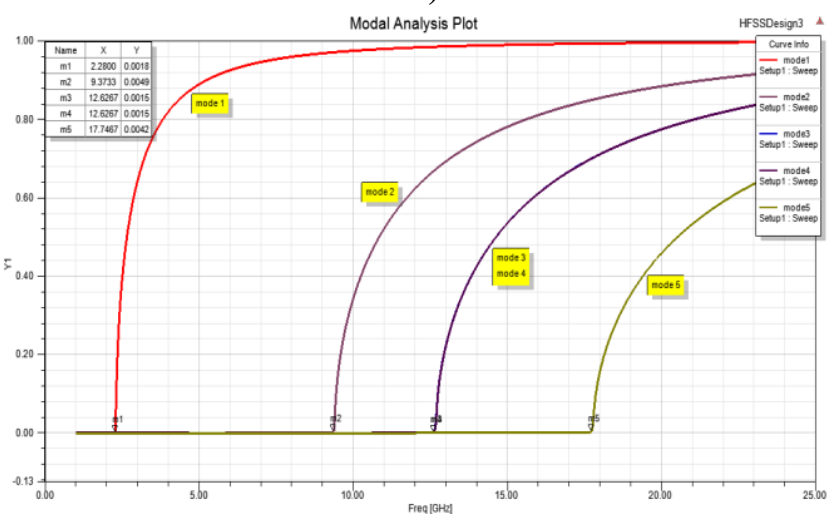

Fig. 9.First Five Modes of Anti-Trapezoidal Waveguide $(\theta=-300)$ 


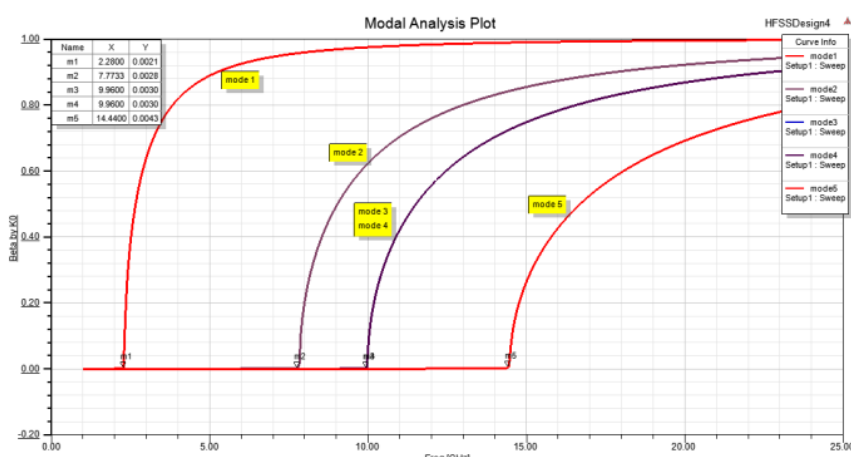

Fig. 10.First Five Modes of Anti-Trapezoidal Waveguide $(\theta=$ - 450)

With reference to the above simulation analysis, the cut-off frequencies of the first four modes of Rectangular, Trapezoidal, Double Ridge and Anti-Trapezoidal waveguides are analyzed and the results are summarized as shown in Table 1.

Table 1. Cut-off frequencies of Rectangular, Trapezoidal, Double Ridge and Anti-trapezoidal waveguides

\begin{tabular}{|l|l|l|l|l|}
\hline Type & \multicolumn{4}{|c|}{ Cut - off Frequencies $(\mathbf{G H z})$} \\
\hline$(\boldsymbol{\theta})$ & mode1 & mode2 & mode3 & mode4 \\
\hline $90^{0}$ & 6.5467 & 13.1067 & 14.7067 & 16.0933 \\
\hline $30^{0}$ & 2.8133 & 15.5067 & 15.5067 & 16.6800 \\
\hline $45^{0}$ & 3.5600 & 15.6667 & 15.6667 & 15.6667 \\
\hline $0^{0}$ & 2.3867 & 12.6800 & 14.7600 & 14.7600 \\
\hline$-30^{0}$ & 2.2800 & 9.3733 & 12.6267 & 12.6267 \\
\hline$-45^{0}$ & 2.2800 & 7.7733 & 9.9600 & 9.9600 \\
\hline
\end{tabular}

The attenuation of these waveguides are also studied and simulated in HFSS and these results also indicate that the specific anti-trapezoidal waveguide provides less attenuation as that of the rectangular, double ridge and trapezoidal waveguide structures. The simulated results are shown below from fig.11-16.

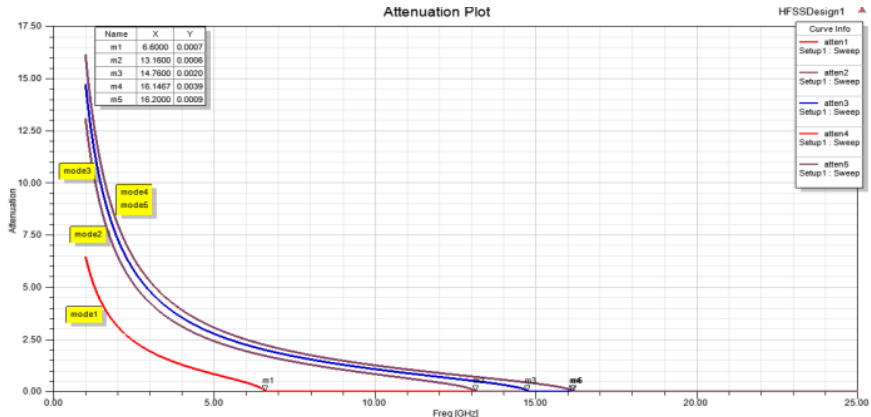

Fig.11. Attenuation Plot of Rectangular Waveguide $(\theta=$

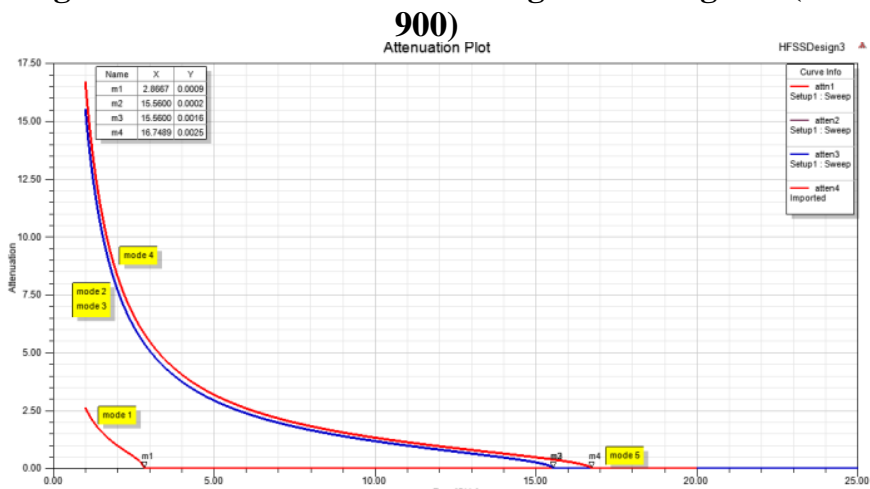

Fig.12. Attenuation Plot of Trapezoidal Waveguide $(\boldsymbol{\theta}=\mathbf{3 0 0})$

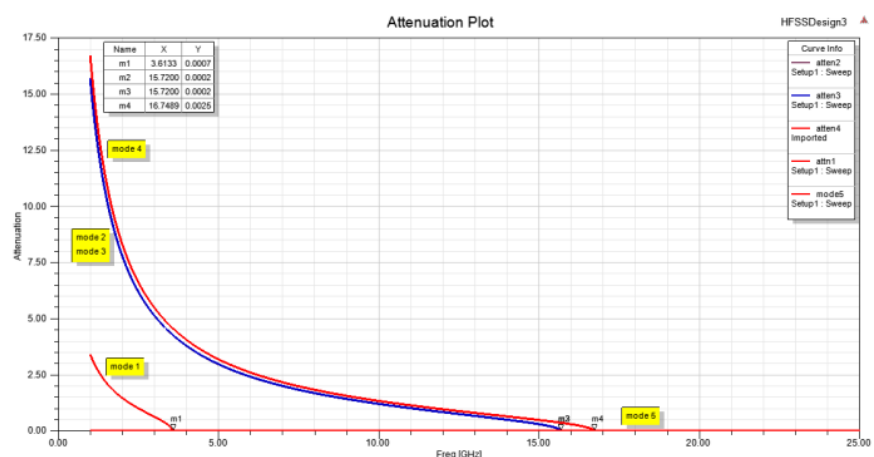

Fig.13. Attenuation Plot of Trapezoidal Waveguide $(\theta=$

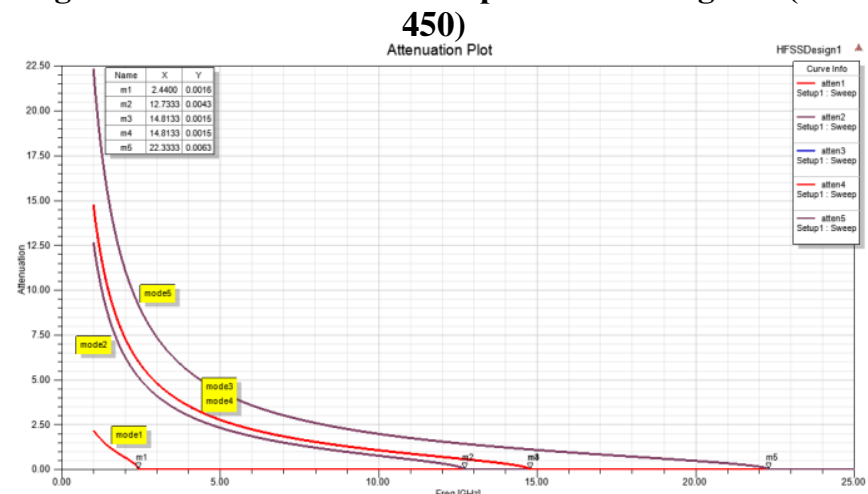

Fig.14. Attenuation Plot of Double Ridge Waveguide $(\theta=$ 00)

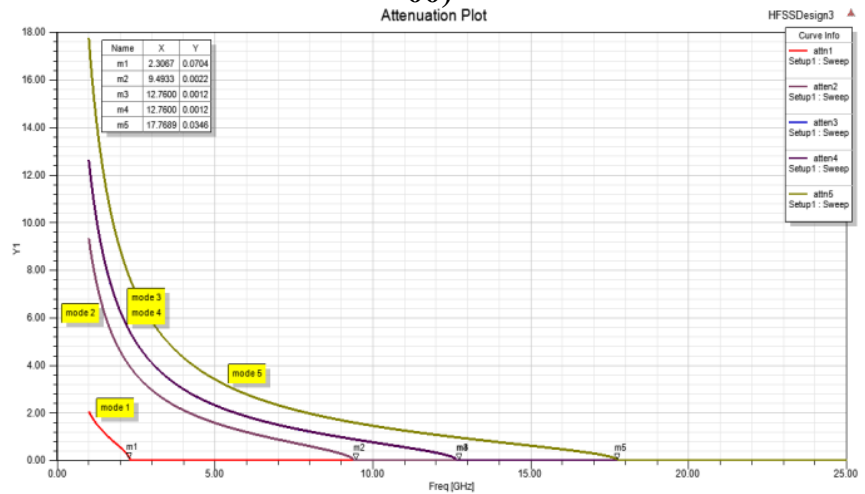

Fig.15. Attenuation Plot of Anti-Trapezoidal Waveguide $(\boldsymbol{\theta}=\mathbf{3 0 0})$

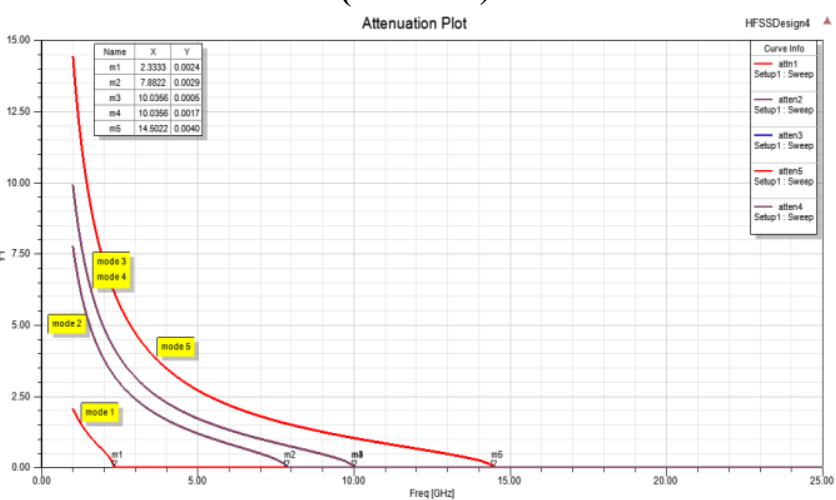

Fig. 16. Attenuation Plot of Anti-Trapezoidal Waveguide

$$
(\boldsymbol{\theta}=\text { - 450) }
$$

All these above results are obtained by maintaining $\mathrm{a} / \mathrm{b}=$ $2.25, \mathrm{~b} / \mathrm{d}=10$ as per industrial standard of WR90 double ridge waveguides except the case of the rectangular waveguide where $\mathrm{a} \mathrm{x} \mathrm{b}$ is same as that of all other waveguides. 


\section{CONCLUSION}

In this paper, we have proposed the anti-trapezoidal waveguides having better performance than that of regular most common rectangular and ridge waveguides. As a primary matter of investigation in our study only cut-off frequencies, bandwidths and attenuations are considered. Further investigation has to be done thoroughly considering other parameters too. For the construction of these waveguides, copper is used as construction material here. Other materials need to be tested in the coming investigation process to reduce the cost and improve the efficiency of these waveguides for mass production as per industry standards. Considering the same dimension for all above waveguides for a particular value of theta will show the lowest cut-off frequency and improved bandwidth. Specific Anti-trapezoidal waveguides, provides better results than the rectangular, double ridge and trapezoidal waveguides.

\section{REFERENCES}

[1] S.H. Hajimowlana, H. Djahanshahi, and M. Kamyab, "Determination of Cut-off Wavelengths of Ridged Waveguides by Modal Analysis," IEEE Proc., MWSCAS, 40 ${ }^{\text {th }}$ ed., Vol. 1, Sacramento, USA, pp. 642-645, August 2002.

[2] S. Hopfer, "The Design of Ridged Waveguides," IEEE Trans. Transactions on Microwave Theory and Techniques, $5^{\text {th }}$ ed., vol. 3, pp. 20-29, October 1955.

[3] Y.Utsumi,"Variational analysis of ridged waveguide modes,"IEEE Trans. Microwave Theory Tech, vol.33(2), pp.111-120,1985.

[4] M. Lu, P.J. Leonard, "Design of trapezoidal-ridge waveguide by finite element method," IEEE Proc., Microw. Antennas Propag., $3^{\text {rd }}$ ed., vol. 151, Bath. UK, pp. 205-211, June 2004

[5] Xiaoqiang Chen, Jie Li, "The Analysis of Attenuation of Trapezoid Ridge Waveguide by Finite Difference Time Domain,",IEEE Proc., IMCE,. Hohhot, China, pp.4494 - 4499, July 2011.

[6] M.F.Iskander, M.A.K.Hamid, "Eigenvalues for a trapezoidal waveguide,",IEEE Proc., The Radio and Electronic Engineer,. $11^{\text {th }}$ ed., vol. 44, Canada., pp.593-596, 1974.

[7] Zhou Ping, Li Qing-bo, "Transmission Characteristics Analysis for Double Trapezoida-ridge Waveguide by the Vector Finite-element Method," IEEE Proc,. iCECE,. vol. 1, Wuhan, China, pp.4510-4512, June 2010 .

[8] C.S. Lee, S.W. Lee, and S.L. Chuang, "Plot of Modal Field Distribution in Rectangular and Circular Waveguides," IEEE Trans. MTT 33, 3 ${ }^{\text {rd }}$ ed., vol. 33, pp. 271-274, Marh 1985.

[9] N. Marcuvitz, Waveguide Handbook. IET, McGraw Hill, 1986.

\section{AUTHORS PROFILE}

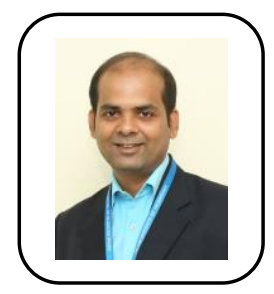

Ramakrushna Panigrahi received the B.Tech Degree in Electronics and Telecommunication Engineering from Biju Patnaik University, Odisha, India in 2009, and the Master Degree in Electronics and Communication Engineering from IIT Kharagpur, West Bengal, India in 2014. He is currently a Assistant Professor with GITAM University, India. He is currently pursuing the Ph.D degree at GITAM University, India. His current research includes ridge waveguides, metallic waveguides and Antenna design for $5 \mathrm{G}$ applications.

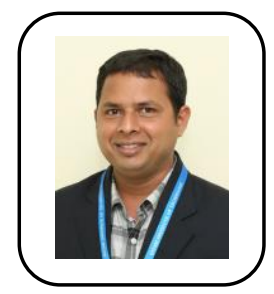

Siba Prasad Mishra received the Bachelor Degree in Electronics and Communication Engineering from Biju Patnaik University, Odisha in 2007, and the Master Degree in Signal Image Professing from NIT Rourkela, Odisha, India in 2014. He is currently a Assistant Professor with GITAM University, India. His current research includes Radar System Design, Signal Optimization in $5 \mathrm{G}$ Networks.

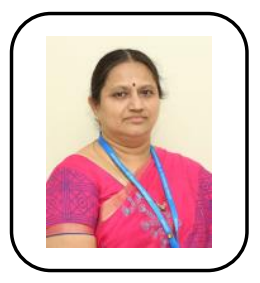

P.V.Y Jayasree received the B.E degree in Electronics and Communication Engineering from GITAM University, Andhra Pradesh, India, in 1989, Master Degree in Electronic Engineering from Andhra University, Vizag, AP, India in 1999, and $\mathrm{Ph} . \mathrm{D}$ degree in EMI EMC from JNTU Kakinada, India in 2010. She is currently Professor, Head of Department of Electrical Electronics and Communication Engineering with GITAM Univeristy, Visakhapatnam, AP, India. She has author or co-author over 32 Journal Papers and 35 conference papers. She has co-authored 7 books. Her current research interests includes antenna design for $5 \mathrm{G}$ applications, tunnel FET, microwave passive circuits and components, low power SRAM cell design, and phased array antennas. Dr. P.V.Y. Jayasree has been the Chair of Board of Studies with GITAM University since 2018 